\title{
Exogenous $v s$. endogenous $\gamma$-glutamyltransferase activity: Implications for the specific determination of S-nitrosoglutathione in biological samples
}

\author{
Emilia Bramanti ${ }^{a}, V$ Valeria Angeli ${ }^{a}$, Maria Franzini ${ }^{b}$, Cecilia Vecoli ${ }^{b}$, Riccardo Baldassini $^{c}$, Aldo Paolicchi $^{c}$, \\ Renata Barsacchi ${ }^{\mathrm{d}}$, Alfonso Pompella ${ }^{\mathrm{c}, *}$ \\ ${ }^{a}$ Italian National Research Council-Istituto per i Processi Chimico-Fisici, Laboratory of Instrumental E Analytical Chemistry, Via G. Moruzzi 1, 56124 Pisa, Italy \\ ${ }^{\mathrm{b}}$ Scuola Superiore Sant'Anna, Piazza Martiri della Libertà 33, 56127 Pisa, Italy \\ ${ }^{\mathrm{c}}$ Department of Experimental Pathology, University of Pisa Medical School, Via Roma 55, 56126 Pisa, Italy \\ ${ }^{\mathrm{d}}$ Department of Biology, Unit of Biochemistry, University of Pisa, Via S. Zeno 51, 56127 Pisa, Italy
}

\section{A R T I C L E I N F O}

\section{Article history:}

Received 23 March 2009

and in revised form 18 May 2009

Available online 23 May 2009

\section{Keywords:}

S-nitrosothiols

S-nitrosoglutathione

S-nitrosocysteinylglycine

$\gamma$-Glutamyltransferase

Liquid chromatography

4,5-Diaminofluorescein

Human plasma

\begin{abstract}
A B S T R A C T
The determination of S-nitrosoglutathione (GSNO) levels in biological fluids is controversial, partly due to the laborious sample handling and multiple pretreatment steps required by current techniques. GSNO decomposition can be effected by the enzyme gamma-glutamyltransferase (GGT), whose involvement in GSNO metabolism has been suggested. We have set up a novel analytical method for the selective determination and speciation of GSNO and its metabolite S-nitrosocysteinylglycine, based on liquid chromatography separation coupled to on-line enzymatic hydrolysis of GSNO by commercial GGT. In a post-column reaction coil, GGT allows the specific hydrolysis of the $\gamma$-glutamyl moiety of GSNO, and the S-nitrosocysteinylglycine (GCNO) thus formed is decomposed by copper ions originating oxidized cysteinylglycine and nitric oxide (NO). NO immediately reacts with 4,5-diaminofluorescein (DAF-2) forming a triazole derivative, which is detected fluorimetrically. The limit of quantitation (LOQc) for GSNO and GCNO in plasma ultrafiltrate was $5 \mathrm{nM}$, with a precision (CV) of 1-6\% within the 5-1500 nM dynamic linear range.

The method was applied to evaluate the recovery of exogenous GSNO after addition of aliquots to human plasma samples presenting with different total GGT activities. By inhibiting GGT activity in a time dependent manner, it was thus observed that the recovery of GSNO is inversely correlated with plasmatic levels of endogenous GGT, which indicates the need for adequate inhibition of endogenous GGT activity for the reliable determination of endogenous GSNO.
\end{abstract}

(c) 2009 Elsevier Inc. All rights reserved.

\section{Introduction}

It is widely accepted that S-nitrosothiols (RSNOs) ${ }^{1}$ are important intermediates in nitric oxide (NO) metabolism, though their significance in pathophysiological phenomena still remains under debate. Several methods have been published in recent years, all including rather aggressive and non-specific decomposition procedures [photolysis, $\mathrm{HgCl}_{2}, \mathrm{HgCl}_{2} / \mathrm{V}(\mathrm{III}), \mathrm{KI} / \mathrm{I}_{2}, \mathrm{Cys} / \mathrm{KI} / \mathrm{Cu}(\mathrm{I})$ etc., liable to cause undesired interactions with components of biological matrices[1]. Besides, a major obstacle in assessing reference values

\footnotetext{
* Corresponding author. Fax: +390502218557.

E-mail address: apompella@biomed.unipi.it (A. Pompella).

1 RSNOs, S-nitrosothiols; GSNO, S-nitrosoglutathione; GGT, gamma-glutamyltransferase; GCNO, S-nitrosocysteinylglycine; LOQc, limit of quantitation; NO, nitric oxide; GG, glycylglycine; LC, liquid chromatography; DAF-2, diaminofluorescein; DMSO, dimethyl sulfoxide; NEM, N-ethylmaleimide; DTPA, diethylentriamine-pentaacetic acid pentasodium salt; SBC, serine/borate complex; DAD, diode array detector; FD, fluorimetric detector.
}

of RSNOs in complex biological matrices is represented by the lack of consolidated procedures for sampling/storage to preserve RSNO stability in the pre-analytical step, as well as of validated analytical methods/protocols and certified reference material [1]. As a consequence, the range of basal RSNO levels reported in various studies has covered more than three orders of magnitude, between low nanomolar (<1-62 nM) and low-micromolar/micromolar levels [1-3].

GGT is a central enzyme for the synthesis and degradation of GSH [4]. GGT can also cause decomposition of GSNO [5,6], and the metabolite GCNO is more susceptible than GSNO to copper ion-catalysed decomposition [7]. Thus, in the presence of glycylglycine (GG) as acceptor, the following reactions occur in vitro:

$$
\begin{aligned}
& \mathrm{GSNO}+\mathrm{GG} \stackrel{\text { GGT }}{\longrightarrow} \mathrm{GCNO}+\gamma \text {-GluGG } \\
& \mathrm{GCNO} \stackrel{\mathrm{Cu}(\mathrm{II})}{\longrightarrow} \mathrm{NO}+\mathrm{GC}_{\mathrm{ox}}
\end{aligned}
$$


It was reported that the reaction of $\mathrm{Cu}(\mathrm{II})$ with $\mathrm{GCNO}$ is fast, with half-lives of about $5 \mathrm{~s}$, while the reaction with GSNO is 3-4 orders of magnitude slower [7]. By in vitro kinetics analysis we have recently shown that bovine kidney GGT has a $K_{\mathrm{m}}$ value for GSNO of $0.398 \pm 0.031 \mathrm{mM}$, comparable with the $K_{\mathrm{m}}$ value for GSH $(0.4 \mathrm{mM})[8]$.

In the present study we have set up a new analytical method for the specific determination and speciation of GSNO and GCNO, based on liquid chromatography (LC) and an on-line, post-column reaction system. In the latter, conditions are adjusted for enzymatic decomposition of GSNO by commercial bovine kidney GGT (reaction (1)), fast decomposition of its product GCNO by copper ions (reaction (2)) [9], and reaction of NO thus formed with 4,5-diaminofluorescein (DAF-2) (reaction (3)) [10,11]:

$\mathrm{NO}+\mathrm{DAF}-2 \longrightarrow$ DAF-2T

The triazole derivative, DAF-2T, is detected and quantified fluorimetrically. The procedure allowed the separation of GSNO and GCNO from compounds present in complex matrices and in the presence of ascorbic acid and uric acid, which are known to interfere with NO detection [12-14]. Besides, data obtained with plasma samples confirmed that a loss of GSNO may occur during pre-analytical handling when endogenous GGT present is not inhibited.

\section{Materials and methods}

\section{Chemicals}

Analytical reagent-grade chemicals were used without further purification. GGT (G-8040, Type I crude from bovine kidney) and glycylglycine (GG, G-1002) were purchased from Sigma-Aldrich (Sigma, Chemical Co., Milan, Italy). Copper sulphate anhydrous (2791) was purchased from Merck (Laborchimica, Firenze, Italy). Five millimolar solution of 4,5-Diaminofluorescein (DAF-2) solution in dimethyl sulfoxide (DMSO) (251510) and its triazole derivative (DAF-2T, 251510, $0.5 \mathrm{mM}$ in DMSO) were purchased from Calbiochem (La Jolla CA, USA).

N-ethylmaleimide (NEM, 04259 BioChemika Ultra, $\geqslant 99.0 \%$ ), DL-serine (84980), boric acid (B-0252) and diethylentriamine-pentaacetic acid pentasodium salt (DTPA, 17969) were purchased from Fluka (Fluka GmbH, Buchs, Switzerland). $50 \mathrm{mM}$ stock solution of ethylenediaminetetracetic acid (405501, EDTA disodium salt, RPE) was purchased from Carlo Erba (Milan, Italy). Phosphate buffer solution (PBS) was prepared from monobasic monohydrate sodium phosphate and dibasic anhydrous potassium phosphate (BDH Laboratory Supplies, Poole, England).

Fresh 0.4 M NEM stock solution was prepared in MilliQ water daily. $0.4 \mathrm{M}$ serine/borate complex (SBC) stock solution was prepared in $1 \mathrm{M}$ PBS $\mathrm{pH}$ 8.0. GGT was prepared in $0.1 \mathrm{M}$ PBS $\mathrm{pH}$ 8.0. $0.2 \mathrm{M}$ GG stock solution was prepared in $1 \mathrm{M}$ PBS pH 8.0. $\mathrm{CuSO}_{4}$ was prepared in MilliQ water.

Stock solution of GSNO (N4148, CAS No. 57564-91-7, Sigma) was prepared in $0.1 \mathrm{M}$ PBS pH 8.0, 0.5 mM DTPA. As such, GSNO standard solution was stable during the working day ( $9 \mathrm{~h}$ time tested at room temperature) and for 4 weeks if stored at $-80^{\circ} \mathrm{C}$.

S-Nitrosocysteinylglycine (GCNO), S-nitrosocysteine (CysNO) and $\mathrm{S}$-nitrosohomocysteine (HCysNO) were prepared by reacting $1 \mathrm{M} \mathrm{NaNO}_{2}$ in $\mathrm{H}_{2} \mathrm{O}$ with $1.1 \mathrm{M}$ thiols in $0.5 \mathrm{M} \mathrm{HCl}, 0.5 \mathrm{mM}$ EDTA at $0{ }^{\circ} \mathrm{C}$ for $40 \mathrm{~min}$ [15]. RSNOs were diluted in $0.1 \mathrm{M}$ PBS pH 8.0, $0.5 \mathrm{mM}$ DTPA and stability was tested before any other use.

Methanol for liquid chromatographic analysis was purchased from Carlo Erba (Rodano, MI, Italy). Water deionized with a Milli-Q system (Millipore, Bedford, MA, USA) was used throughout.

\section{Spectrophotometric analysis}

Concentrations of GSNO, CysNO, HCysNO and GCNO in $0.1 \mathrm{M}$ PBS, pH 8.0, at $21 \pm 0.1{ }^{\circ} \mathrm{C}$ were calculated from the absorbance at $334 \mathrm{~nm}$ [16] by a Beckman DU-600 spectrophotometer.

\section{Fluorimetric analysis}

A fluorescence spectrophotometer (Perkin-Elmer LS-45 Luminescence Spectrophotometer) was used for method optimization. Slit width was $10 \mathrm{~nm}$ for both excitation $\left(\lambda_{\mathrm{ex}}=485 \mathrm{~nm}\right)$ and emission $\left(\lambda_{\mathrm{em}}=515 \mathrm{~nm}\right)$. Measurements were performed in $1 \mathrm{~mL}, 1 \mathrm{~cm}$ path length cuvette. The concentration of DAF-2 standard solution used for determinations was optimized between 0.025 and $0.5 \mu \mathrm{M}$. The commercial product DAF-2T gave a calibration curve linear in the range $1-20 \mathrm{nM}$ (slope $=74.4 \pm 1.7, R=0.9992, N=5$ ) and a detection limit of $1 \mathrm{nM}$.

\section{Measurement of GGT activity}

GGT activity was determined by following the hydrolysis of $\gamma$-glutamylcarboxy- $p$-nitroanilide (Glu-cp-NA) as previously described [17]. Briefly, purified enzyme was incubated with $1 \mathrm{mM}$ Glu-cp-NA and $20 \mathrm{mM}$ GG in PBS $(0.1 \mathrm{M}, \mathrm{pH} 8.0)$ at $37^{\circ} \mathrm{C}$ and the absorbance increase at $405 \mathrm{~nm}$ was monitored. Enzyme activity was determined using a molar absorption coefficient of $8800 \mathrm{M}^{-1} \mathrm{~cm}^{-1}$ for the formation of $p$-nitroaniline.

\section{Human blood sampling and storage}

Plasma aliquots were derived from routine clinical samples obtained from 13 different in-patients (Inst. of Clinical Physiology-CNR, Pisa). Venous blood was collected by venipuncture using EDTA as anticoagulant. After low-speed centrifugation (1500 g, $10 \mathrm{~min}$ ) at room temperature, plasma samples were stored at $-20^{\circ} \mathrm{C}$ until analysis. One milliliter of plasma was immediately spiked with $10 \mu \mathrm{L}$ of $50 \mathrm{mM}$ DTPA stock solution $(0.5 \mathrm{mM}$ final concentration of DTPA in plasma) and $30 \mu \mathrm{L}$ of $0.4 \mathrm{M}$ NEM stock solution (12 mM final concentration of NEM). Then, GGT inhibitor serine/borate complex (SBC; $10 \mathrm{mM}$ final concentration) was added before GSNO spike or after various intervals incubation time. The addition of DTPA/NEM/SBC solution avoided losses of GSNO due to transnitrosation reactions, metal- and enzyme-mediated decomposition (see Results).

Before analysis, plasma samples were diluted 1:1 in $0.1 \mathrm{M}$ PBS $\mathrm{pH} 7.4,0.5 \mathrm{mM}$ EDTA, loaded onto the sample reservoir of an Amicon Microcon YM-3 centrifugal filter units (cut-off $3000 \mathrm{Da}$; Millipore, Bedford, MA, USA) and centrifuged at $11000 \mathrm{~g}$ for 90 min at $4{ }^{\circ} \mathrm{C}$, to remove proteins and high molecular weight compounds. Ultrafiltrates were then injected in the chromatographic system. For the experiments of recovery of spiked RSNO in human whole blood, RSNOs were added after the addition of DTPA/NEM/ SBC solution.

\section{Instrumental set-up}

The HPLC unit consisted of a pump (P4000, ThermoQuest) equipped with a Rheodyne 7125 injector (Rheodyne, Cotati, CA, USA), a $100 \mu \mathrm{L}$ poly(etheretherketone) (PEEK tubing, Upchurch, Oak Harbor,WA) injection loop and an HPLC column (Gemini RP $\mathrm{C}_{18} 250 \times 4.6 \mathrm{~mm}$, silica particle size $5 \mu \mathrm{m}$, equipped with a Guard Cartridge KJ0-4282, Phenomenex). The eluent for the chromatographic analysis was 99\% 0.02 M PBS pH 6.0/1\% methanol (99:1 $\mathrm{v} / \mathrm{v}$ ), flowing in the optimized conditions at $0.8 \mathrm{~mL} / \mathrm{min}$. The flow eluting from HPLC column was monitored between 210 and $650 \mathrm{~nm}$ by a UV-visible diode array detector (DAD) equipped with 


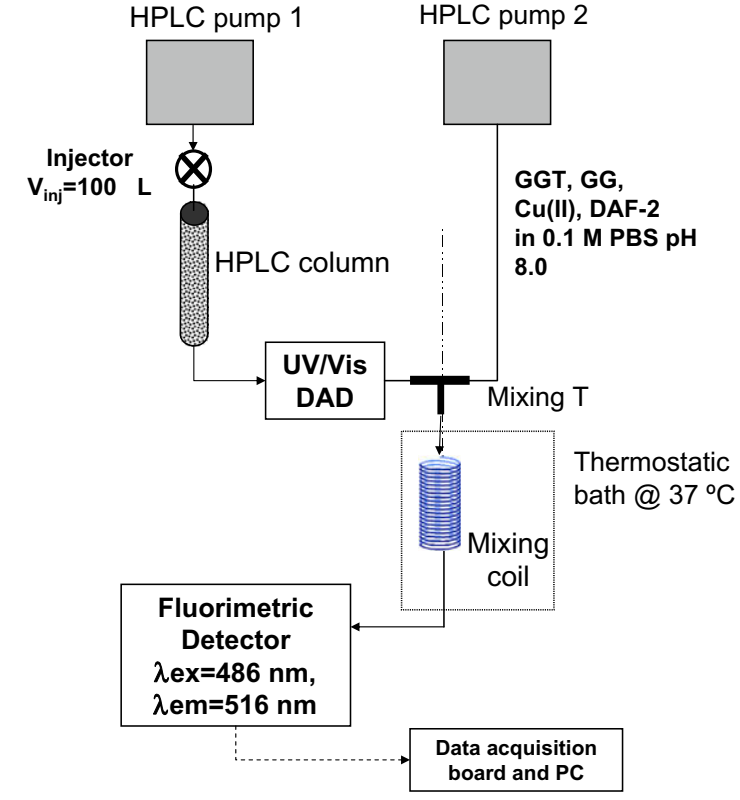

Fig. 1. Schematic diagram of LC-FD system for the determination of GSNO and other RSNOs.

a $5 \mathrm{~cm}$ path length flow cell (UV6000, ThermoQuest). The effluent was blended in a low dead-volume mixing-tee with the reaction solution delivered by a second HPLC pump (Jasco PU2080) at a flow rate of $0.3 \mathrm{~mL} / \mathrm{min}$, containing $140 \mathrm{mU} / \mathrm{mL} \mathrm{GGT,} 0.03 \mathrm{mM}$ copper sulphate, $1.2 \mathrm{mM} \mathrm{GG}$ and $0.37 \mu \mathrm{M}$ DAF-2 in $0.1 \mathrm{M} \mathrm{PBS}$ pH 8.0 (optimized conditions, concentrations in the reaction coil). The enzymatic reaction proceeded in the optimized conditions in a PFA reaction coil of $2.8 \mathrm{ml}$, corresponding to a $t_{\text {reaction }}=2.55 \mathrm{~min}$, kept at the constant temperature of $37^{\circ} \mathrm{C}$ in a water bath (Fig. 1). Thus, the DAF-2T signal was specifically detected by a fluorescence detector (FL3000, ThermoFinnigan) operating at excitation wavelength $\lambda_{\mathrm{ex}}=486 \mathrm{~nm}$ and emission wavelength $\lambda_{\mathrm{em}}=516 \mathrm{~nm}$. Fig. 1 shows a detailed schematic of the LC-DAD-FD system equipped with the post-column reaction system described.

\section{Results}

\section{UV spectrophotometric experiments}

UV spectrophotometry was used to roughly optimize GG, GGT and $\mathrm{CuSO}_{4}$ concentrations, prior to optimizing on line reaction conditions (see below). Optimization was performed by a Box-Wilson central composite design, varying GG in the range 5-20 mM, GGT in the range $300-1200 \mathrm{mU} / \mathrm{mL}$, and $\mathrm{CuSO}_{4}$ between 50 and $250 \mu \mathrm{M}$. Experiments were run in $1 \mathrm{~mL}$ cuvettes, in $0.1 \mathrm{M} \mathrm{PBS} \mathrm{pH}$ 8.0. GG, copper and GGT were added at the established concentrations in the order reported above. Thus, the blank was read in the spectrophotometer, and GSNO was added as last component before starting kinetics data acquisition. By varying the concentration of GGT and GG, and by evaluating the time-dependent plots of the absorbance at $334 \mathrm{~nm}$, we found that $525 \mathrm{mU} / \mathrm{mL}$ GGT and 4.5 mM GG (final concentrations) were the optimal conditions for the quantitative decomposition ( $\geqslant 95 \%$ ) of GSNO in about $300 \mathrm{~s}$ at $37^{\circ} \mathrm{C}$. In the absence of copper and chelating agents, transition metals present as contaminants in the reaction medium decomposed GCNO slowly. Increasing concentrations of copper rapidly decomposed GCNO. When the reaction medium also included EDTA, GCNO decomposition was not prevented, provided that copper was added in excess with respect to EDTA. A concentration of
$100 \mu \mathrm{M} \mathrm{Cu}$ (II) was sufficient for the quantitative decomposition of $100 \mu \mathrm{M}$ GSNO in $300 \mathrm{~s}$, in the presence of $525 \mathrm{mU} / \mathrm{mL}$ GGT and $4.5 \mathrm{mM} \mathrm{GG}$. The same experiments performed in the absence of GGT showed that copper by itself (up to $1.2 \mathrm{mM}$ ) did not decompose GSNO in the 300 s observation time (data not shown).

In the operating conditions employed ( $4.5 \mathrm{mM} \mathrm{GG}, 0.1 \mathrm{mM}$ $\mathrm{CuSO}_{4}, 525 \mathrm{mU} / \mathrm{mL} \mathrm{GGT}$ ), the stability of three other S-nitrosothiols was also verified, GCNO, HcysNO, and CysNO. In the absence of GGT GSNO was stable, GCNO was instantaneously decomposed, as previously reported [9], CysNO was instantaneously and fully decomposed as well, and HCysNO was decomposed by about $75 \%$ (data not shown).

\section{Fluorimetric experiments}

GGT-catalysed/copper-mediated GSNO hydrolysis was then investigated in cuvette by fluorescence spectrometry, in order to optimize the DAF-2-mediated detection of the product NO. Experiments were run in $1 \mathrm{~mL}$ cuvette in $0.1 \mathrm{M}$ PBS pH 8.0. GG, copper sulphate, GGT and DAF-2 were added at the established concentration. Thus, blank was obtained $\left(\lambda_{\mathrm{ex}}=485 \mathrm{~nm}, \lambda_{\mathrm{em}}=515 \mathrm{~nm}\right)$ and GSNO was then added as last component, before starting the kinetics data acquisition.

The GGT-catalysed/copper-mediated decomposition of $0.3 \mu \mathrm{mol} / \mathrm{L}$ GSNO obtained with five different concentrations of DAF-2 $(0.5,0.3,0.1,0.05$ and $0.025 \mu \mathrm{M}$ DAF-2) showed an increase of fluorescence signal as a function of time, which corresponded to formation of DAF-2T following the release of NO from GCNO (data not shown). The reaction was complete for a reaction time $t \geqslant 5 \mathrm{~min}$ for all the concentrations of DAF-2 considered, as expected on the basis of spectrophotometric kinetics at $334 \mathrm{~nm}$. At increasing concentrations of DAF-2 the plateau value $F_{10}$ (=the fluorescence signal after 10 min reaction time) increased as well, indicating an increase of NO detection sensitivity obtained at higher DAF-2 concentrations. However, the overlapping of curves obtained with 0.5 and $0.3 \mu \mathrm{M}$ DAF- 2 clearly indicated that above $0.3 \mu \mathrm{M}$ DAF-2 the rate of reaction (3) no longer depends on DAF2 concentration, but likely on the rate of formation of the intermediate $\mathrm{N}_{2} \mathrm{O}_{3}[10,11]$. At higher DAF-2 concentrations (e.g. $0.5 \mu \mathrm{M}$ ) the blank signal obtained in the absence of GSNO increased as well, thus affecting the baseline noise signal. Thus, the sensitivity factor (i.e. the slope of the calibration curve) and the baseline noise, which is correlated with the concentration detection limit (LODc), depend on the DAF-2 concentration chosen for the analysis.

Table 1 summarizes the results of data fitting of calibration curves of GSNO standard solutions performed in cuvette in $0.1 \mathrm{~mol} \mathrm{~L}^{-1} \mathrm{PBS} \mathrm{pH} 8.0$, using three different concentrations of DAF-2 $(0.05,0.1$ and $0.5 \mu \mathrm{M})$. The LODc of the method in cuvette was $5 \mathrm{nM}$, and the dynamic linear range was $15-200 \mathrm{nM}$. It was verified that in the absence of GGT the fluorescence signal did not increase.

Optimization of the reaction conditions for the determination of GSNO and GCNO in plasma

Although GGT specificity makes the proposed approach applicable to in-batch measurements of GSNO, the presence in complex biological matrices (e.g. plasma) of other RSNOs also susceptible to copper-mediated decomposition would require a double determination - with/without GGT - in order to determine (by subtraction) the specific GSNO signal. On the other hand, the set up of a chromatographic separation - coupled to an on line post-column reaction system including reactions ()$(1)-(3)$ - would make it possible to speciate GSNO as well as other RSNOs susceptible to copper-mediated decomposition. Furthermore, by choosing suitable separation conditions the interference by compounds known 
Table 1

Fitting parameters of the linear part of GSNO calibration curves obtained at three different DAF-2 concentrations (50, 100 and $500 \mathrm{nM})$.

\begin{tabular}{llllll}
\hline [DAF-2] $(\mathrm{nM})$ & Slope $\left(\right.$ Volts $\left.\mathrm{nM}^{-1}\right)$ & Intercept (Volts) & $N$ & $R$ & DLR $^{\mathrm{a}}(\mathrm{nM})$ \\
\hline 50 & $1.41 \pm 0.09$ & $10.0 \pm 5.7$ & 7 & 0.9966 & $15-200$ \\
100 & $2.66 \pm 0.09$ & $15.1 \pm 2.2$ & 8 & 0.9948 & $15-200$ \\
500 & $4.87 \pm 0.05$ & $66.7 \pm 2.5$ & 7 & 0.9961 & $15-200$ \\
\hline
\end{tabular}

${ }^{a}$ Dynamic linear range.

to affect the formation of DAF-2T, such as uric, ascorbic and dehydroascorbic acids [12-14], would be controlled.

Thus, the HPLC separation/post-column reaction procedure was adapted and optimized for GSNO analysis in complex biological matrices. Firstly, the HPLC apparatus coupled to the post-column reaction system and fluorimetric detector (FD) was operated in flow injection mode (=without HPLC column). A solution of commercial GSNO was injected in order to optimize detection conditions: concentration of GGT acceptor GG (ranging 1-20 mmol/L), $\mathrm{Cu}$ (II) $(100-800 \mu \mathrm{mol} / \mathrm{L})$, DAF-2 $(0.1-2 \mu \mathrm{mol} / \mathrm{L})$, GGT $(0.2-1.5 \mathrm{U} /$ $\mathrm{mL}$ ), as well as temperature of the thermostatic bath.

Subsequently, HPLC/post-column reaction system/FD in the normal configuration mode (=with the HPLC column inserted between the injection valve and the DAD, Fig. 1), was used to optimize the flow rate of HPLC eluent and post-column reaction solution, and the length of the PFA reaction coil. Parameters were chosen in order to maximize the GSNO signal and minimize the peak width at half height $\left(W_{1 / 2}\right)$. This was obtained (i) by limiting the post-column dilution of reactants, and (ii) by increasing the post-column reaction time. A 2.7:1 ratio between HPLC eluent flow and post column solution flow gave optimal results. A flow of $0.8 \mathrm{~mL} / \mathrm{min}$ was suitable for the separation of GSNO and the other RSNOs (see below). A flow of $0.3 \mathrm{~mL} / \mathrm{min}$ was chosen for the postcolumn reaction. Fig. 2A shows the LC-FD chromatograms obtained by injecting a fixed concentration of GSNO without post-column reaction coil or with PFA reaction coils of various lengths, corresponding to increasing reaction times (considering a total flow in the reaction coil of $1.1 \mathrm{~mL} / \mathrm{min}$ ). Fig. $2 \mathrm{~B}$ shows the normalized slope of GSNO calibration curve (left $y$ axis) and the $W_{1 / 2}$ values (right $y$ axis) as a function of the reaction time.

The slope values of calibration curves reflected the kinetics obtained in experiments in cuvette. $W_{1 / 2}$ values were linear as a function of reaction time, as expected. We chose a reaction coil of $2.8 \mathrm{~mL}$, which gave a good sensitivity factor (GSNO was decomposed by GGT by about $65-70 \%$ ) and a $W_{1 / 2}$ value compatible with HPLC separation of all RSNOs studied.

Table 2 summarizes values of all post-column reaction parameters, optimized for GSNO determination by LC-DAD-FD. Concentrations shown are those in the reaction coil when considering a 3.7 dilution factor, due to the mixing of HPLC flow $(0.8 \mathrm{~mL} / \mathrm{min})$ with post-column reaction solution $(0.3 \mathrm{~mL} / \mathrm{min})$.

As discussed above, $\mathrm{Cu}(\mathrm{II})$ can rapidly decompose also CysNO and HCysNO. Thus, in the optimized conditions listed in Table 2, the four RSNOs (GSNO, GCNO, CysNO and HCysNO) could be efficiently determined by the method proposed. Fig. 3 shows the LC-FD chromatogram of a mixture of CysNO, HCysNO, GCNO and GSNO solutions. We verified that sodium nitrite and nitrate solutions injected at $\mathrm{mM}$ concentrations gave no fluorescence signal (not shown)

Further determinations were performed in presence or absence of GGT in order to verify the selectivity of GGT reaction as to GSNO. In the absence of GGT, GSNO gave a peak about 1/4 of the one obtained in the presence of GGT (data not shown), indicating some degree of not GGT-related GSNO decomposition. This effect may be produced by $\mathrm{Cu}(\mathrm{II})$ being simultaneously present in the reaction medium with GG (required as acceptor substrate for GGT) and
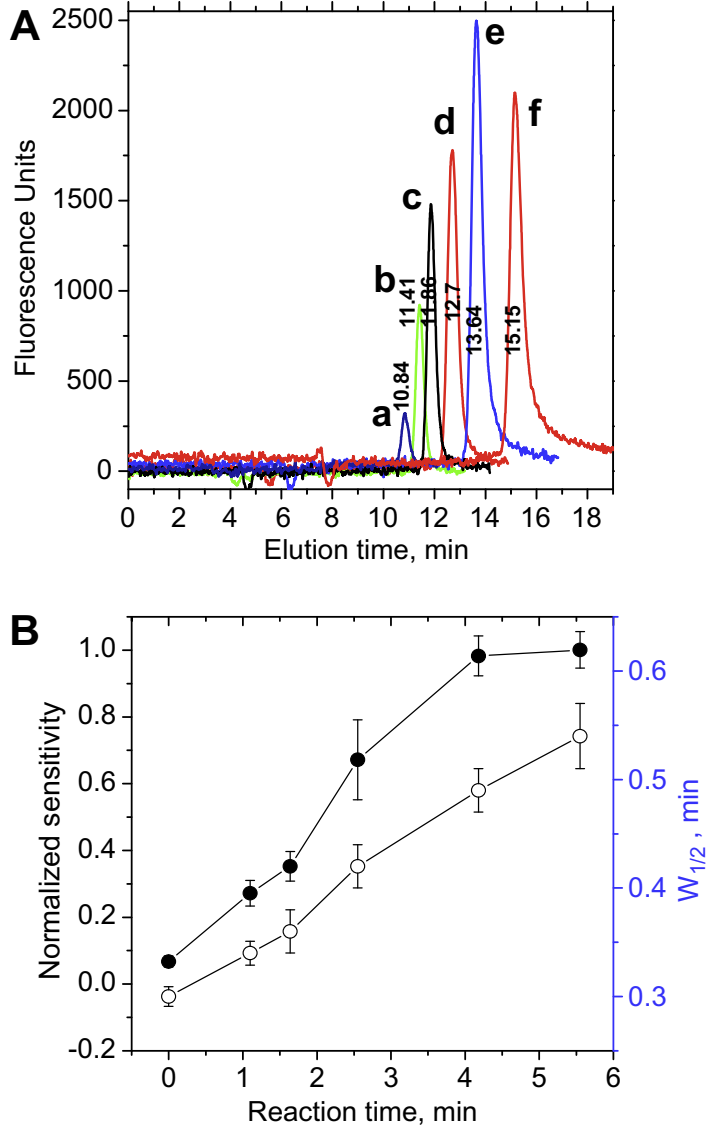

Fig. 2. Optimization of GGT/Cu++/DAF-2-mediated GSNO determination. (A) LC-FD chromatograms obtained by injecting a $330 \mathrm{nM}$ GSNO with no post-column reaction coil or PFA reaction coils of various length: (a) no reaction coil, (b) $1.2 \mathrm{~mL}$, (c) $1.8 \mathrm{~mL}$, (d) $2.8 \mathrm{~mL}$, (e) $4.6 \mathrm{~mL}$, (f) $6.1 \mathrm{~mL}$. Operating conditions: $1.2 \mathrm{mM}$ GG, $0.37 \mu \mathrm{M}$ DAF-2, $140 \mathrm{mU} / \mathrm{mL}$ GGT, $0.03 \mathrm{mM} \mathrm{Cu}(\mathrm{II})$, HPLC flow $0.8 \mathrm{~mL} / \mathrm{min}$, postcolumn reactant $0.3 \mathrm{~mL} / \mathrm{min}$ (concentrations in the reaction coil). (B) Normalized slope of GSNO calibration curve (left $y$ axis) and $W_{1 / 2}$ values (right $y$ axis) as a function of the post-column reaction time.

DAF-2. It is known in fact that formation of adducts with various ligands, including amino acids [18] and DAF-2 itself [16,17], can alter the redox potential of transition metals, often making them more reactive.

\section{Recovery of GSNO from plasma}

Fig. 4 shows typical LC-FD and UV absorbance chromatograms of plasma ultrafiltrates. Spikes of RSNOs were added to blood after

Table 2

Optimized values of all parameters for the determination of GSNO and RSNO by HPLC coupled to the post-column reaction system and fluorometric detector.

\begin{tabular}{ll}
\hline Parameter & Optimized value \\
\hline GG concentration & $1.2-4.5 \mathrm{mM}^{\mathrm{a}}$ \\
Cu(II) concentration & $0.03-0.250 \mathrm{mM}$ \\
DAF-2 concentration & $0.37 \mu \mathrm{M}$ \\
GGT concentration & $140 \mathrm{mU} / \mathrm{mL}$ \\
HPLC flow & $0.8 \mathrm{~mL} / \mathrm{min}$ \\
Post-column reaction solution flow & $0.3 \mathrm{~mL} / \mathrm{min}$ \\
PFA reaction coil length & $2.8 \mathrm{ml}$ \\
Temperature of the thermostatic bath & $37-4{ }^{\circ} \mathrm{C}$ \\
\hline
\end{tabular}

a We indicated a range for several parameters that can be varied without affecting significantly the $S / N$ ratio. The sensitivity factor has to be calculated in the chosen reaction conditions. 


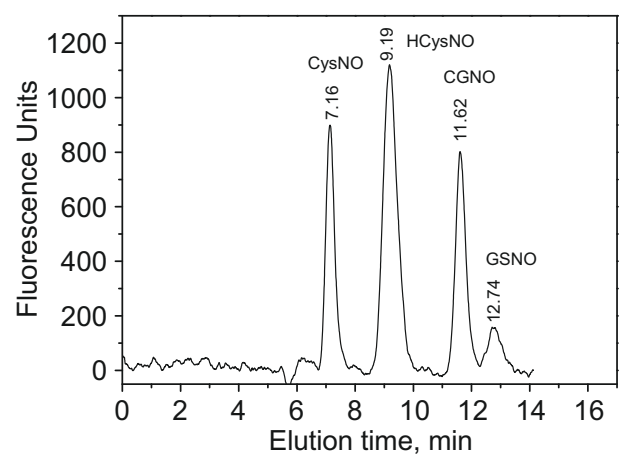

Fig. 3. Determination of different RSNOs by the GGT/Cu++/DAF-2 method. LC-FD chromatograms of CysNO, HCysNO, GCNO and GSNO obtained by using the $2.8 \mathrm{~mL}$ reaction coil: $115 \mathrm{nM}$ CysNO, $350 \mathrm{nM}$ HCysNO, $80 \mathrm{nM}$ GCNO and $30 \mathrm{nM}$ GSNO.

addition of the stabilizing NEM/DTPA/SBC solution, required to avoid losses due to transnitrosation reactions and metal- and/or GGT-mediated GSNO decomposition.

Table 3 summarizes the results of fitting of RSNOs calibration curves obtained in buffer and analyte addition curve in plasma, in the operating conditions of Table 2 , in the $5-1500 \mathrm{nM}$ concentration range. Recovery experiments of RSNOs spiked in plasma evidenced a good recovery and linear analyte addition curves for GSNO (98.6\%) and GCNO (99.5\%) in the 5-1500 nM range. The limit of quantitation (LOQC) of GSNO and GCNO of the proposed method was $5 \mathrm{nM}$, with a precision (CV\%) of $1.4-5.7 \%$ for GSNO and $1-5.8 \%$ for GCNO within the dynamic linear range (5-1500 nM) at $0.37 \mu \mathrm{M}$ DAF-2 concentration in the post-column reaction coil. LOQc was calculated on the basis of the signal to noise ratio of baseline noise and the slope of calibration curves, and determined experimentally by analysing GSNO and GCNO.

Recoveries of CysNO and HCysNO were instead significantly lower (40-60\%) and analyte addition curves were exponential, showing lower recoveries at lower concentrations (5-50 nM) and higher recoveries at higher concentrations (50-1500 nM). Furthermore, the LOQc value determined experimentally for both CysNO and HCysNO was $20 \mathrm{nM}$. Such low recoveries of CysNO and HCysNO may be due to interference with NO/DAF-2 reaction by ascorbic, dehydroascorbic and uric acids [12-14], which eluted simultaneously to CysNO (ascorbic and dehydroascorbic acid) and HCysNO (uric acid) (Fig. 4), taking into account the shift due to post-column reaction time.

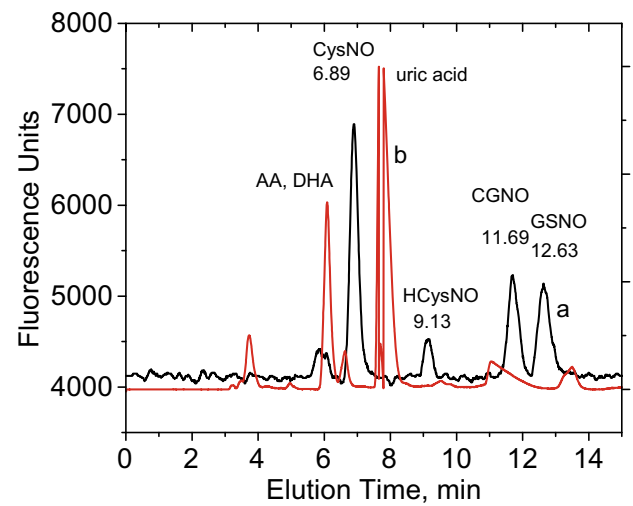

Fig. 4. Recovery of different RSNOs exogenously added to plasma. LC-DAD-FD chromatograms (trace $a$ ) and UV absorbance chromatogram at $254 \mathrm{~nm}$ (trace $b$ ) of a plasma ultrafiltrate sample obtained from blood spiked with $600 \mathrm{nM}$ CysNO, $150 \mathrm{nM}$ HCysNO, $100 \mathrm{nM}$ GCNO and $180 \mathrm{nM}$ GSNO. RSNO concentration found was $310 \mathrm{nM}$ CysNO (recovery 51\%), 70vnM HCysNO (recovery 47\%), $106 \mathrm{nM}$ GCNO (recovery 106\%) and $170 \mathrm{nM}$ GSNO (recovery 94\%).
Table 3

Fitting parameters of RSNO calibration curves in $0.1 \mathrm{M} \mathrm{PBS} \mathrm{pH} 8,0.5 \mathrm{mM}$ DTPA and analyte addition curves in plasma in the optimized operating conditions ofTable 2.

\begin{tabular}{lrlll}
\hline RSNO & $t_{\mathrm{R}}(\mathrm{min})$ & Slope (f.u. ${\left.\mathrm{min} \mathrm{nM}^{-1}\right)}^{2}$ & $R^{2}$ & $N$ \\
\hline Buffer & & & & \\
GSNO & 12.74 & $2.77 \pm 0.002$ & 0.9999 & 10 \\
CNOG & 11.62 & $4.16 \pm 0.005$ & 0.9999 & 10 \\
CysNO & 7.16 & $2.80 \pm 0.002$ & 0.9999 & 10 \\
HCysNO & 9.19 & $1.91 \pm 0.005$ & 0.9999 & 10 \\
& & & & \\
Plasma & & & & \\
GSNO & 12.63 & $2.73 \pm 0.011$ & 0.9999 & 9 \\
CNOG & 11.69 & $4.16 \pm 0.005$ & 0.9999 & 7 \\
CysNO & 6.89 & Not linear & - & - \\
HCysNO & 8.13 & Not linear & - & - \\
\hline
\end{tabular}

Recovery experiments of RSNOs spiked in plasma (approx. $100 \mathrm{nM}$ final conc.) showed that GSNO and GCNO were stabile in plasma stored at $-80{ }^{\circ} \mathrm{C}$ up to 3 days (recoveries $\geqslant 85 \%$ ), while after 1 week the recovery was $\approx 50 \%$. Spiked CysNO and HCysNO were unstable: after $24 \mathrm{~h}$ the recovery was less than $50 \%$. In plasma ultrafiltrate RSNOs were stable only during the working day. Thus, while GSNO and GCNO can be accurately determined in plasma ultrafiltrate by the LC-FD method, separation and detection of CysNO and HCysNO is not possible in plasma, though potentially feasible in simpler matrices.

\section{Hydrolysis of GSNO by endogenous plasmatic GGT}

The good separation of GCNO and GSNO, their good recoveries and determination limit in plasma allowed us to correlate the stability of spiked GSNO with the endogenous content of GGT. In all blood samples used, DTPA and NEM were added before GSNO spike. The metal chelator DTPA should avoid RSNOs losses due to metal-catalysed decomposition [9]. Alkylation by NEM is able to block the reactivity of - $\mathrm{SH}$ groups in about $20 \mathrm{~s}$ at neutral $\mathrm{pH}$ at room temperature [19]. The addition of NEM to plasma samples should therefore prevent artifactual formation of RSNOs due to transnitrosation reactions from proteins (basically nitroso-albumin) [20] and thiols (in particular GSH) present at micromolar concentration levels in plasma [21]. Moreover, if not blocked by NEM, reduced thiols could reduce adventitious metals present in biological samples: in particular, $\mathrm{Cu}(\mathrm{I})$ originating from reduction of $\mathrm{Cu}$ (II) could catalyse the decomposition of GSNO [7]. Fig. 5 shows an example of LC-FD chromatogram of human plasma spiked with $60 \mathrm{nM}$ GSNO, and analysed after allowing increasing times before GGT inhibition (=addition of SBC: at $0,1,5$ and $10 \mathrm{~min}$ ). $T=0$ was evaluated by adding SBC before the GSNO spike.

As can be seen, when increasing times were allowed before addition of SBC to plasma samples, proportionally lower GSNO recoveries were obtained, while amounts of detectable GCNO correspondingly increased.

From the areas of chromatographic peaks of GCNO $\left(t_{\mathrm{R}}=11.90 \mathrm{~min}\right)$ and GSNO $\left(t_{\mathrm{R}}=12.73 \mathrm{~min}\right)$ and their respective calibration curves, in each sample it was possible to determine at different times the concentrations of residual GSNO and GCNO originated by GGT activity (reaction (1)). Data indicate that depending on the addition time of GGT inhibitor SBC, recoverable GSNO progressively decreased, along with a corresponding increase of GCNO. Fig. 6 shows data obtained with four representative plasma samples (out of 13 examined), characterized by different GGT activities. RSNO concentration was normalized with respect to the GSNO concentration added $(60 \mathrm{nM})$. At $t=0$ the recovery of GSNO was quantitative $(97 \pm 5 \%)$, while at later times GSNO was converted into GCNO more and more quickly, in correlation with increasing plasma GGT levels. 


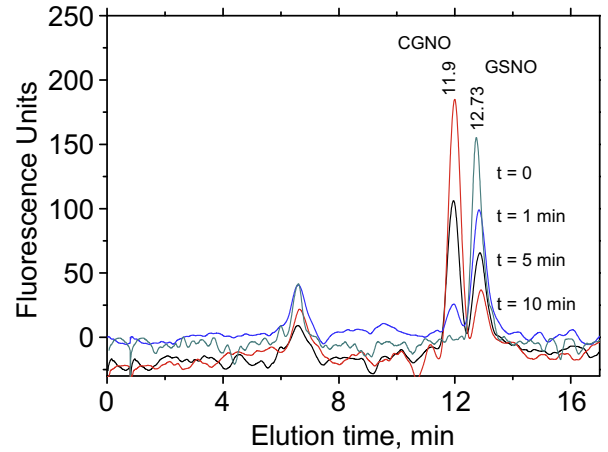

Fig. 5. Interference of endogenous GGT with GSNO determination (I). LC-FD chromatograms of human plasma stabilized by NEM/DTPA, spiked with $60 \mathrm{nM}$ GSNO, and analysed after allowing increasing times before GGT inhibition (=addition of SBC: at $0,1,5$ and $10 \mathrm{~min}$ ). $T=0$ was evaluated by adding the SBC before the GSNO spike. The sample shown contained $114 \mathrm{U} / \mathrm{L}$ total GGT. GSNO recovery at $t=0$ was here 93\% taking into account the 1:1 dilution factor of plasma before ultracentrifugation.

Fig. 7 shows the percentage of residual GSNO found in 13 plasma samples after 5 and $10 \mathrm{~min}$ with respect to the total GSNO spiked, as a function of plasma GGT activity.

An inverse correlation was observed between plasma GGT activities and percentages of recovered GSNO (linear correlation parameters after $5 \mathrm{~min}$ : intercept $87 \pm 4 \%$; slope $=-0.18 \pm 0.02$;

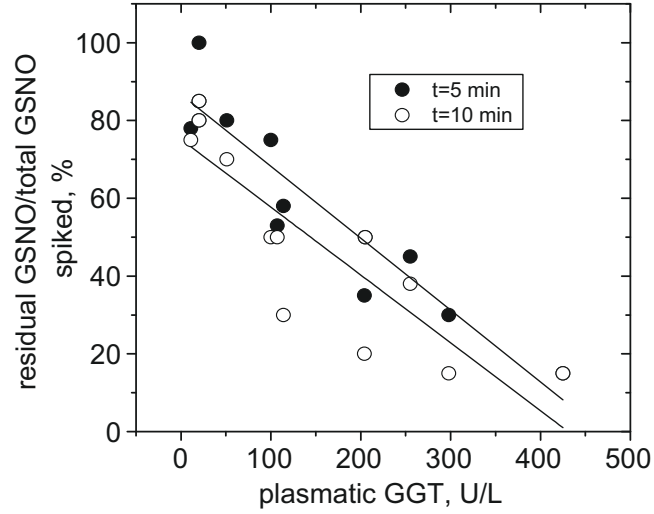

Fig. 7. Interference of endogenous GGT with GSNO determination (III). Recovery of GSNO after 5 (closed circles) and 10 min GGT inhibition time (open circles), as a function of basal GGT activities present in plasma. Recovery was reported as percentage of the concentration of GSNO found over the total amount spiked. Linear correlation parameters after $5 \mathrm{~min}$ : intercept $87 \pm 4 \%$; slope $=-0.18 \pm 0.02$; $R^{2}=0.8696$; linear correlation after $10 \mathrm{~min}$ : intercept $75 \pm 6 \%$; slope $=-0.17 \pm 0.03$; $R^{2}=0.7410$.

$\left.R^{2}=0.8696\right)$. A positive correlation was found, instead, between plasma GGT activity and the formation of GCNO (linear correlation parameters after $10 \mathrm{~min}$ : slope $\left.=0.23 \pm 0.03 ; R^{2}=0.8021\right)($ data not shown). The concentration ([GSNO] + [GCNO]) over 10 min was not
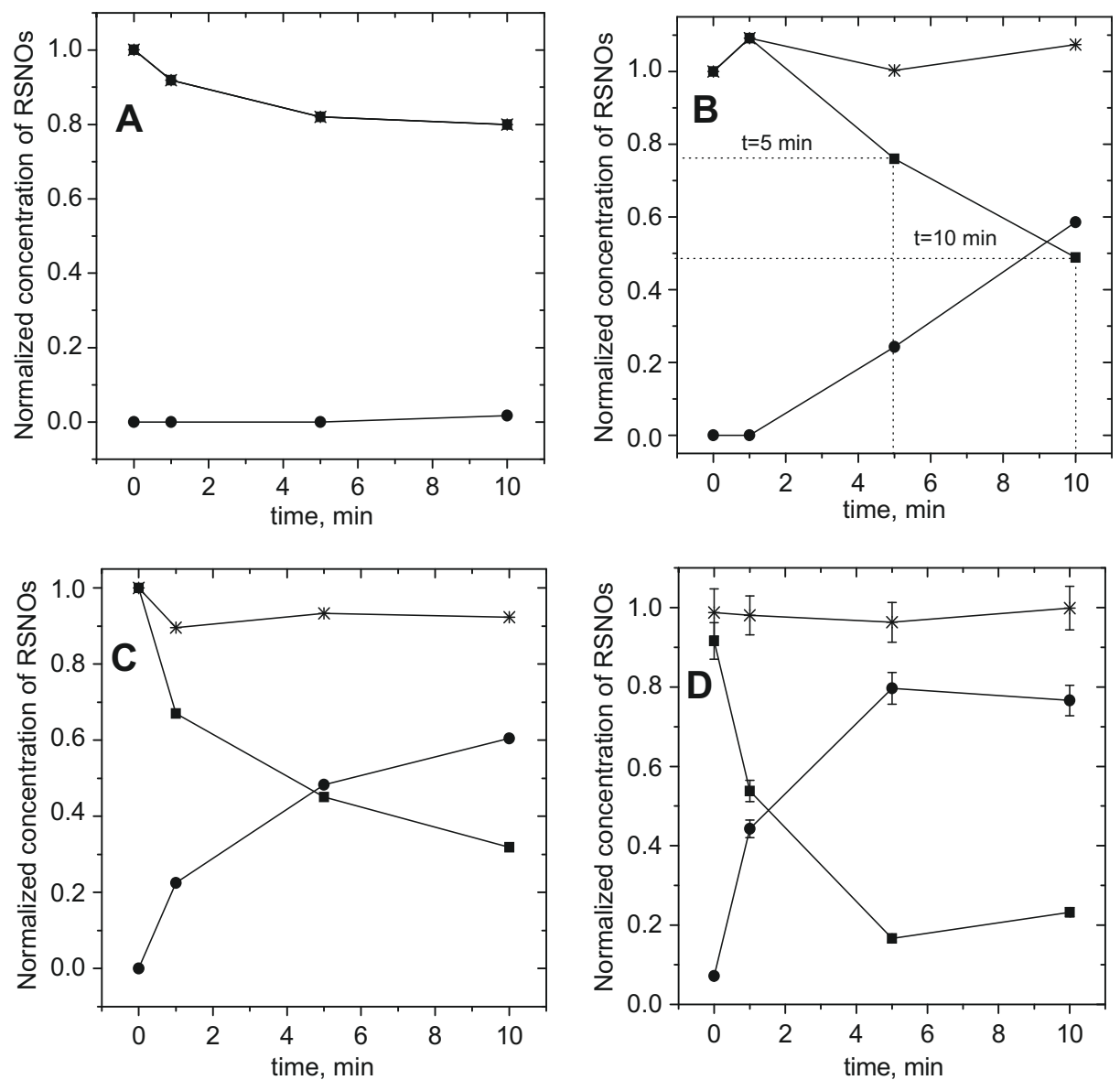

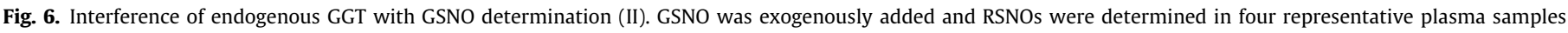

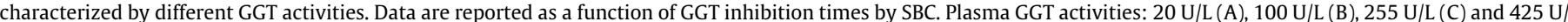

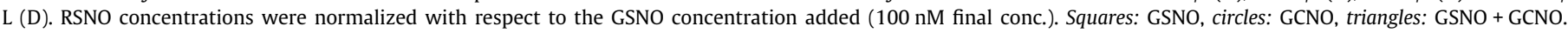
Representative error bars (SD) are reported in panel $\mathrm{D}(N=3$ replicates). 
significantly different from the concentration of spiked GSNO, thus indicating a quantitative conversion of GSNO into GCNO.

\section{Discussion}

The results of the present study highlight a fundamental aspect to be considered in GSNO research, i.e. the patho-physiological role of endogenous GGT activity possibly (and variably) present in samples. Data reported in a previous paper from our laboratory, concerned with the kinetics of GGT with respect to GSNO as a substrate and glycylglycine as acceptor co-substrate [8], suggested that plasmatic GGT can be a factor in catabolism of GSNO in blood, where numerous endogenous, physiological acceptor co-substrates are available. Altogether our observations are of high relevance as far as GSNO determination: if - besides the inhibition of transnitrosation reactions and metal-induced decomposition - endogenous GGT activity is not efficiently inhibited during sampling (pre-analytical step), the analyte may be lost during the following 1-10 min, directly depending on the levels of GGT present in plasma. This observation may explain the elusiveness of GSNO determination reported in the literature, despite the significant methodological advances achieved over the recent years.

Thus, SBC at the indicated concentration of $10 \mathrm{mM}$ should be used during blood sampling in order to avoid GGT-related GSNO loss, as previously envisaged [22]. Immediate and efficient GGT inhibition is however a more complex issue than apparent. SBC aliquots should perhaps be prepared in ad hoc sampling vessels, possibly of the vacuum-type. Using the present method or another recently published by us [22], plasma GSNO concentrations were found to range in between 30 and $300 \mathrm{nM}$; these data are however to be regarded as preliminary, and subject to confirmation. Moreover, a further aspect liable to affect determination is given by possible haemolysis: even minimal levels could in fact contribute unpredictable amounts of erythrocytic GSNO to the measured values. Studies are in progress in our laboratory in order to investigate these points comprehensively.

In conclusion, a liquid chromatographic system coupled with a post column injection system delivering a buffer solution containing GGT, GG, copper and DAF-2 allowed us to fluorimetrically determine and speciate GSNO and other RSNOs in plasma ultrafiltrate. The idea of using GGT as a reagent to promote the on line hydrolysis of GSNO into GCNO, which in turn is labile to coppermediated decomposition, represents a novel approach to the long debated analytical problem of specific GSNO determination in biological samples. GSNO and GCNO were determined with a LOQc of $5 \mathrm{nM}$, a precision (CV) of $1-6 \%$ in the $5-1500 \mathrm{nM}$ dynamic linear range, and a recovery $98.6 \%$ (GSNO) and $99.5 \%$ (GCNO) in plasma ultrafiltrate. The method allowed the appreciation of a critical aspect never investigated before, i.e. the direct interference of (endogenous) GGT activity with detectable GSNO levels. Last but not least, the method has the advantage of using reagents by far less toxic or environmentally hazardous as compared to $\mathrm{HgCl}_{2}$ salts, generally used in current procedures for RSNO decomposition in the 0.1-0.2 $\mathrm{mM}$ concentration range.

\section{Conflict of interest}

Authors C.V., Aldo P., R.B., M.F., A.P. and E.B. have patented the described procedures (Patent No. PCT/IB2007/002090, July 2007). Authors Aldo P., M.F., A.P. and E.B. are members in a University Spin-off company (Sorta Srl., www.sorta-biomedical.com) devoted to contract research based on different analytical services, including the one presented here.

\section{Acknowledgments}

This work has been financially supported by the Italian National Research Council-CNR and the University of Pisa. Additional funds were derived from the Fondazione per lo Studio della Fibrosi Cistica (grant: FFC 11/2005; Verona, Italy) and Istituto Toscano Tumori (ITT, Firenze, Italy; Grant 2008).

\section{References}

[1] D. Giustarini, A. Milzani, R. Colombo, I. Dalle-Donne, R. Rossi, Trends Pharmacol. Sci. 25 (2004) 311-316.

[2] P.H. MacArthur, S. Shiva, M.T. Gladwin, J. Chromatogr. B 851 (2007) 93-105.

[3] D. Giustarini, A. Milzani, I. Dalle-Donne, R. Rossi, J. Chromatogr. B 851 (2007) 124-139.

[4] A. Pompella, A. Visvikis, A. Paolicchi, V. De Tata, A.F. Casini, Biochem. Pharmacol. 66 (2003) 1499-1503.

[5] S.C. Askew, A.R. Butler, F.W. Flitney, G.D. Kemp, I. Megson, Bioorg. Med. Chem. 3 (1995) 1-9.

[6] N. Hogg, R.J. Singh, E. Konorev, J. Joseph, B. Kalyanaraman, Biochem. J. 323 (1997) 477-481.

[7] A.P. Dicks, H.R. Swift, D.L.H. Williams, A.R. Butler, H.H. Al-Sadoni, B.C. Cox, J. Chem. Soc., Perkin Trans. 2 (1996) 481-487.

[8] V. Angeli, A. Tacito, A. Paolicchi, R. Barsacchi, M. Franzini, R. Baldassini, C. Vecoli, A. Pompella, E. Bramanti, Arch. Biochem. Biophys. 481 (2009) 191-196.

[9] D.R. Noble, D.L.H. Williams, Nitric Oxide 4 (2000) 392-398.

[10] H. Kojima, N. Nakatsubo, K. Kikuchi, S. Kawahara, Y. Kirino, H. Nagoshi, Y. Hirata, T. Nagano, Anal. Chem. 70 (1998) 2446-2453.

[11] N. Nakatsubo, H. Kojima, K. Kikuchi, H. Nagoshi, Y. Hirata, D. Maeda, Y. Imai, T. Irimura, T. Nagano, FEBS Lett. 427 (1998) 263-266.

[12] X. Zhang, W.-S. Kim, N. Hatcher, K. Potgieter, L.L. Moroz, R. Gillette, J.V. Sweedler, J. Biol. Chem. 277 (2002) 48472-48478.

[13] V.S. Kim, X. Ye, S.S. Rubakhin, J.V. Sweedler, Anal. Chem. 78 (2006) 1859-1865.

[14] T. Suzuki, Nitric oxide 16 (2007) 266-273.

[15] A. Doctor, R. Platt, M.L. Sheram, A. Eischeid, T. McMahon, T. Maxey, J. Doherty, M. Axelrod, J. Kline, M. Gurka, A. Gow, B. Gaston, Proc. Natl. Acad. Sci. USA 102 (2005) 5709-5714.

[16] W.R. Mathews, S.W. Kerr, J. Pharmacol. Exp. Toxicol. 267 (1993) 1529-1537.

[17] A. Meister, S.S. Tate, O.W. Griffith, Methods Enzymol. 77 (1981) 237-253.

[18] D.M. Miller, G.R. Buettner, S.D. Aust, Free Rad. Biol. Med. 8 (1990) 95-108.

[19] J.D. Gregory, J. Am. Chem. Soc. 77 (1955) 3922-3923.

[20] O. Rafikova, R. Rafikov, E. Nudler, Proc. Natl. Acad. Sci. USA 99 (2002) $5913-$ 5918.

[21] M.A. Mansoor, A.M. Svardal, P.M. Ueland, Anal. Biochem. 200 (1992) 218-229.

[22] E. Bramanti, K. Jacovozzi, L. D’Ulivo, C. Vecoli, R. Zamboni, Z. Mester, A. D’Ulivo, Talanta 77 (2008) 684-694. 